\title{
The linkage between stimulus frequency and covert peak areas as it relates to monaural localization
}

\author{
MEGAN E. ROGERS and ROBERT A. BUTLER \\ University of Chicago, Chicago, Illinois
}

\begin{abstract}
Head-related transfer functions for differently centered narrow noise bands were obtained on 6 subjects. Derived from these measurements were covert peak areas (CPAs), defined as the spatial constellation of loudspeakers that generates maximal sound pressure at the entrance of the ear canal for specific bands of frequency. On the basis of previous data, we proposed that different frequency bands served as important spectral cues for monaural localization of sounds from different loci and that location judgments were directed toward the CPAs associated with the different bands. In the first study, the stimuli were bandpass filtered so that they contained only those frequencies whose associated CPAs occupied either the monaural listener's "upper" or "lower" spatial regions. Loudspeakers, separated by $15^{\circ}$, were stationed in the left hemifield, ranging from $0^{\circ}$ to $180^{\circ}$ azimuth and $-45^{\circ}$ to $60^{\circ}$ elevation. Subjects reported the loudspeaker from which the sound appeared to originate. Judgments of the sound's elevation were in general accord with the CPAs associated with the different frequency segments. In the second study, monaural localization tests were administered in which different $2.0-\mathrm{kHz}$-wide frequency bands linked with specific CPAs were notch filtered from a $3.5-\mathrm{kHz}$ highpass noise band. For the control condition, the highpass noise was unfiltered. The data demonstrated that filtering a frequency segment linked with specific CPAs resulted in significantly fewer location responses directed toward that particular spatial region. These results demonstrate in greater detail the relation between the directional filtering properties of the pinna and monaural localization of sound.
\end{abstract}

Although binaural localization of sound in space is consistently more accurate than monaural localization, listeners are able to localize sounds in the lateral regions of the hemifield with a fair degree of accuracy when using only one ear. A reasonable explanation of this observation is that people simply learn what an acoustic stimulus sounds like when it comes from a particular region in space. Durlach and Colburn (1978) were more specific. They proposed that we learn the acoustic receptivity pattern of our own pinnae and that we also become familiar with the spectra of everyday sounds. The sound's spectrum at the entrance of the ear canal is the result of the external sound's spectrum being modified by the directional filtering properties of the pinna. Note, however, that according to Durlach and Colburn, proficient monaural localization requires prior knowledge of the spectrum of the sound source. This idea, although attrac-

This research was supported in part by NIH Grant R01 NS25889-02. The authors wish to express their gratitude to Victoria Cefaratti and Michael Umlauf for their assistance in collecting some of the data. Alan Musicant was most helpful in providing technical assistance and suggestions on the preparation of the manuscript; Biao Zhang served as a statistical consultant. Both authors are in the departments of Surgery and Psychology at the University of Chicago. Correspondence should be addressed to R. A. Butler, Otolaryngology-Head and Neck Surgery, University of Chicago Medical Center, Box 412, 5841 S. Maryland Ave., Chicago, IL 60637. tive, is most difficult to test empirically. What, for example, constitutes a familiar sound? Theoretically, knowledge of the listener's acoustic history would be requiredan improbable attainment. Even if a great deal of spectral overlap for sound is normally encountered in everyday experience, a large library of templates would have to be available in order to match an incoming complex sound with the appropriate one previously established in the central auditory nervous system (Carlile, 1990).

Although only in principle can one rule out learning as the sole determinant of monaural localization of sound in space, it may be of some value to consider possible innate mechanisms that could underlie monaural location judgments. Of interest is the phenomenon that frequency has a spatial referent. Studies have shown that when listening monaurally (or binaurally when sound originates in the median sagittal plane), the apparent location of a tone or narrow noise band is strongly influenced by its frequency content, not its actual position in space. Blauert (1969), for example, presented listeners with 1/3-octave bands originating from either in front or behind. When the center frequency of the band was set at $4.0 \mathrm{kHz}$, listeners showed a strong tendency to report the sound as originating in front; at center frequencies of $8.0 \mathrm{kHz}$, the sound appeared to come from overhead; and at center frequencies around $12.0 \mathrm{kHz}$, the sound was usually reported to come from behind, then from the front when the center frequency reached $16 \mathrm{kHz}$. That the frequency 
composition of narrow noise bands governs, in large part, their apparent location was confirmed and extended by other investigators (Butler \& Flannery, 1980; Hebrank \& Wright, 1974; Musicant \& Butler, 1984). The powerful influence of tonal frequency on the apparent location of sound in the median sagittal plane was reported earlier by Pratt (1930) and elaborated upon by Roffler and Butler (1968). At first glance, this phenomenon is an anomalyan auditory illusion. Yet, the fact of its ubiquity warrants further research into its functional significance.

In what way might the spatial referent of stimulus frequency be critical for monaural localization? To begin with, the spatial referent of a frequency segment can be defined both behaviorally and physically. Behaviorally, it is the place in space that the listener identifies as the origin of the narrow noise band. Physically, it is the spatial location of the narrow noise band that generates a maximum amplitude when measured at the ear canal entrance. Normally, response amplitude is plotted against stimulus frequency, with stimulus location serving as the parameter. The peak of the function is the point of greatest amplitude in the frequency response curve. But in the frequency range where the pinna contours modify the sound's spectrum, that is, $>4.0 \mathrm{kHz}$, the frequency response curve nearly always peaks at about 4.0-6.0 kHz, irrespective of the location of the sound source. Hence, in this form, the peak in the response function may only provide spectral information on sounds originating from a relatively restricted region. More informative is the plot of response amplitude as a function of source location, with stimulus frequency serving as the parameter. The peak of the latter function has been referred to as the covert peak-"covert" in the sense that it is not apparent for the higher audio frequencies when response amplitude is plotted against stimulus frequency (Butler, 1987). Yet, this peak has a special property, namely, it is the frequency segment that is boosted more when the sound originates from one particular location than it is from any other location in space. ${ }^{1}$ In short, the covert peak of a particular frequency segment is linked to a specific region in space in that the frequency segment emanating for that spatial region generates a maximum sound pressure at the ear canal entrance. Rather than confining the spatial referent of a narrow frequency band, defined physically, to the point that generates maximum sound pressure, we have arbitrarily defined the spatial referent to be the region in space from which a narrow frequency band generates a sound pressure within $1 \mathrm{~dB}$ of the maximum pressure recorded. In this paper we refer to this spatial region as the covert peak area (CPA). In the animal literature and more recently in the human literature, what we refer to as a CPA is called an isolevel contour. Isolevel contours have been constructed to illustrate the frequencydependent directional properties of the pinna in humans (Middlebrooks, Makous, \& Green, 1989) and in other mammals, for which there is a wealth of data. Musicant, Chan, and Hind (1990) as well as Carlile (1990) point out the parallels in the spectral transformations of the pinna among humans and several other mammalian species. Our CPA can be considered as a 1-dB isolevel contour. Musicant and Butler (1984) demonstrated that for a narrow noise band, centered at a given frequency and presented in the horizontal plane, the spatial referent defined behaviorally and that defined physically were in close accord with one another.

The present study further investigates the adequacy of frequency segments with their associated CPAs as major cues for monaural localization. We calculated the CPAs in a group of listeners for a range of differently centered 1.0-kHz-wide noise bands. With the obtained information on the spatial locations of the CPAs associated with different frequency bands, we conducted two series of localization tasks. In the first, we used two noise bands tailored for each listener such that the CPA associated with one of the bands was located above (in the vertical plane) the CPA associated with the other band. We asked whether each listener's monaural location judgments for each frequency band would lie within the bands' respective CPAs. In the second study, we notched out different bands of frequency from a $3.5-\mathrm{kHz}$ highpass noise. Our question was a simple one: Would monaural listeners be less likely to report the sound as coming from the spatial region that was, in physical terms, the CPA linked to the frequency segment that was now notched out? The spatial distribution of the listeners' monaural location responses when an unfiltered sound was the stimulus served as the basis for comparison.

\section{METHOD}

Six listeners, who had been tested extensively on monaural localization of broadband noise, participated in the study. Their auditory thresholds were within $10 \mathrm{~dB}$ of audiometric zero for frequencies from 0.25 to $8.0 \mathrm{kHz}$. All measurements, physical and behavioral, were carried out in a room $4.8 \times 4.1 \times 2.4 \mathrm{~m}$. The floor was carpeted, a heavy curtain hung behind the loudspeaker array, and the walls were covered by loose felt contained within a fine wire mesh. The distance between the wire mesh and the solid walls was $10 \mathrm{~cm}$.

\section{Physical Measurements}

Head-related transfer functions. Each subject's left external ear canal was occluded by the insertion of a malleable plastic substance (Audi-Sil); a miniature microphone (Knowles Model EA 1934) was embedded in the substance with its diaphragm facing outward. $\mathrm{He} / \mathrm{she}$ was then brought into the test room, seated in a chair equipped with a headrest, and instructed to face straight ahead $\left(0^{\circ}\right.$ in the horizontal and vertical planes) and not to move his/her head during the presentation of a lengthy series of stimuli. He/she was previously informed about the procedure and purpose of the measurement sessions. Noise bands, $1.0 \mathrm{kHz}$ wide and centered at $4.0-14.0 \mathrm{kHz}$ in steps of $0.5 \mathrm{kHz}$, were delivered singly at a fixed level by each of 104 loudspeakers (Realistic Midrange Tweeters, Model 40-1289A) chosen for comparable frequency response characteristics from a pool of 220 . Additionally, a parametric equalizer (Rane Model PE-15) was utilized to approximate a flat spectrum up to $15.0 \mathrm{kHz}$. The stimuli were generated by modulating a 0.5 $\mathbf{k H z}$ lowpass noise with a sinusoid whose frequency defined the center frequency of the noise band. The loudspeakers, supported by pipes bent to specification, were stationed in the subject's left hemifield. They were positioned equidistant $(1.3 \mathrm{~m})$ from the 
listener's head and arrayed from $0^{\circ}$ to $180^{\circ}$ in the horizontal plane and from $-45^{\circ}$ to $60^{\circ}$ in the vertical plane, with $0^{\circ}$ elevation corresponding to the level of the interaural axis. Within each row and each column, the loudspeakers were separated by $15^{\circ}$ center to center. The output of the microphone was amplified (HewlettPackard Model 465A) and fed to a voltmeter (B\&K Type 2409) from which the readings in decibels were recorded. A session consisted of 2,184 measurements-21 differently centered noise bands delivered by 104 loudspeakers. With the center frequency fixed, we recorded the output from the microphone when systematically activating the loudspeakers row by row. Normally, a measurement session lasted from 2 to $2 \frac{1 / 2}{\mathrm{~h}}$. Four complete sets of measurements were collected for each subject. With the output of the loudspeakers remaining unchanged, four complete sets of measurements were also carried out when the microphone was placed in the space formerly occupied by the subject's left ear. These measurement sessions were interspersed with those taken from the subject's ear canal because we wanted to be certain that no changes in the output of the microphone occurred during the prolonged period over which the measurements were taken. And, indeed, no systematic changes did occur. For each subject, we programmed a computer to calculate the median decibel value of the microphone's output for each center frequency delivered by each loudspeakers. From these median values, we subtracted the respective median values of the microphone measurements in space for each differently centered band of noise delivered by each of the 104 loudspeakers. Since all values were in decibels, subtracting one set of measurements from the other was tantamount to dividing the data from the microphone in the ear canal by the data from the microphone in space. The final result was our estimate of the spectral components for each subject that, when combined, would have defined a head-related transfer function for each of the 104 loudspeaker locations. We interpreted these values as the pinna's response to different sounds coming from different spatial locations.

Extracting CPAs. For each subject, we scanned through his/her pinna response starting with the $1.0-\mathrm{kHz}$-wide noise band centered at $4.0 \mathrm{kHz}$. We located the loudspeaker position among the 104 that generated the maximal amplitude relative to free field. Next, we identified all other loudspeaker positions that when activated by the same stimulus generated an output from the microphone within $1 \mathrm{~dB}$ of the maximum output. We refer to this constellation of loudspeakers as the CPA for the $1.0-\mathrm{kHz}$-wide noise band centered at $4.0 \mathrm{kHz}$. We calculated, for each subject, the CPA for each differently centered 1.0-kHz-wide noise band. In all, CPAs for noise bands centered at $4.0-14.0 \mathrm{kHz}$, in $0.5-\mathrm{kHz}$ steps, were identified. These were plotted individually on a $13 \times 8$ matrix -13 positions in the horizontal plane and 8 positions in the vertical plane. Some examples taken from rectangular plots and drawn on unit spheres are shown in Figure 1. Each sphere represents the left hemifield of the listener with a loudspeaker placed at each horizontal-vertical intersection. The listener is facing $0^{\circ}$ azimuth. In this figure, loudspeaker positions comprising the CPAs for the narrow bands centered at $6.0,9.0$, and $11.0 \mathrm{kHz}$ are illustrated for 3 listeners, V.C., Z.M., and M.R. Filled circles designate the position of those loudspeakers whose output was within $1 \mathrm{~dB}$ of the maximum output for the 6.0-kHz-centered noise band. The open circles designate those loudspeakers whose output was within $1 \mathrm{~dB}$ of peak output for the 9.0-kHz-centered noise band; the open triangles designate those loudspeakers whose output was within $1 \mathrm{~dB}$ of maximum output for the noise band centered at $11.0 \mathrm{kHz}$. Although not apparent in Figure 1, CPAs for the lower center frequencies covered a more extensive region-an observation consonant with the isolevel contours published by Middlebrooks et al. (1989). To attain a broader view of each listener's spatial distribution of the CPAs as a function of frequency content of the noise bands, we calculated the mean of the horizontal plane coordinates and the mean of the vertical plane coordinates of the CPAs for each differently centered frequency. Take, for example, the CPA calculated for the $6.0-\mathrm{kHz}$-centered
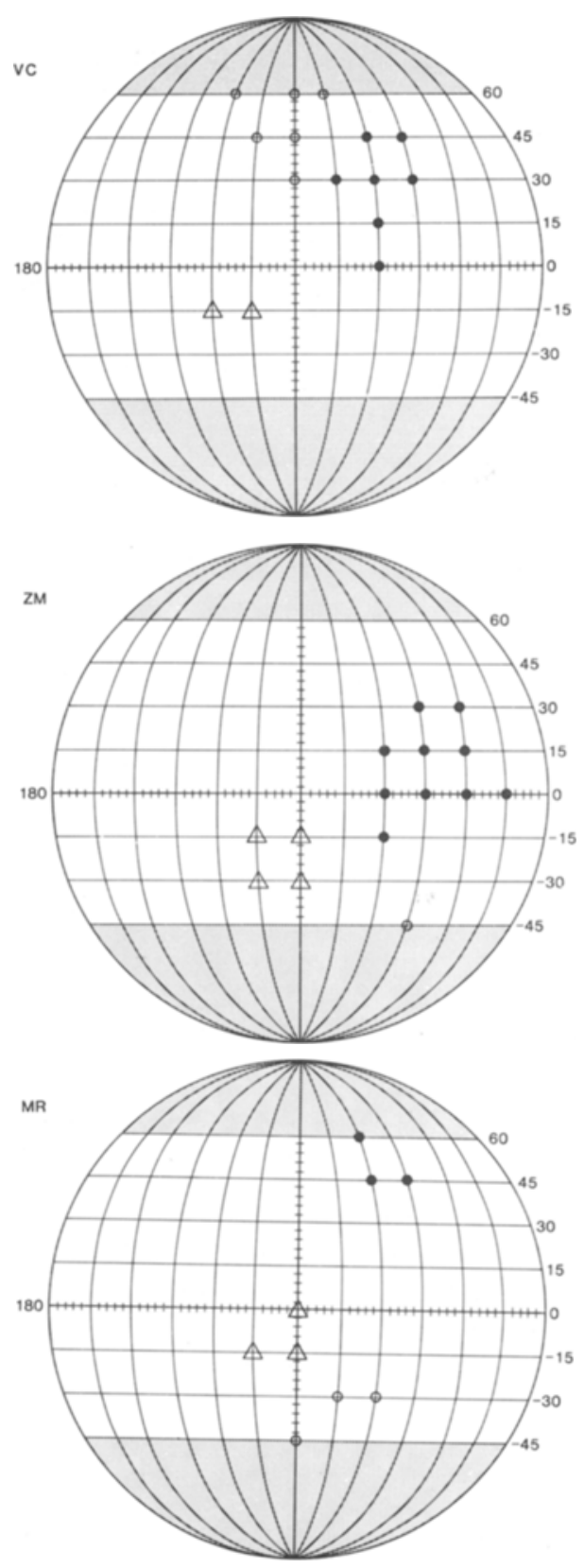

Figure 1. CPAs plotted for 3 listeners (designated by initials) associated with 1.0-kHz-wide noise bands centered at $6.0 \mathrm{kHz}$ (filled circles), $9.0 \mathrm{kHz}$ (open circles), and $11.0 \mathrm{kHz}$ (open triangles).

narrow noise band measured in the ear canal entrance of Listener V.C. The output of 6 of the 104 microphones was within $1 \mathrm{~dB}$ of that loudspeaker (the $7 \mathrm{th}$ ) which generated the maximum output measured at the ear canal entrance. Two of these loudspeakers were positioned at $45^{\circ}$ azimuth, 4 at $60^{\circ}$ azimuth, and 1 at $75^{\circ}$ azimuth. The mean horizontal location of this group of loudspeakers was $58^{\circ}$. With respect to their location in the vertical plane, 1 loudspeaker was located at $0^{\circ}$ elevation, 1 at $15^{\circ}$ elevation, 3 at $30^{\circ}$ elevation, and 2 at $45^{\circ}$ elevation. The mean vertical location of this loudspeaker group was $28^{\circ}$. The mean value of the CPA associated with the $1.0-\mathrm{kHz}$-wide noise band centered at $6.0 \mathrm{kHz}$ for Listener V.C. is plotted in Figure 2 together with the spatial loca- 

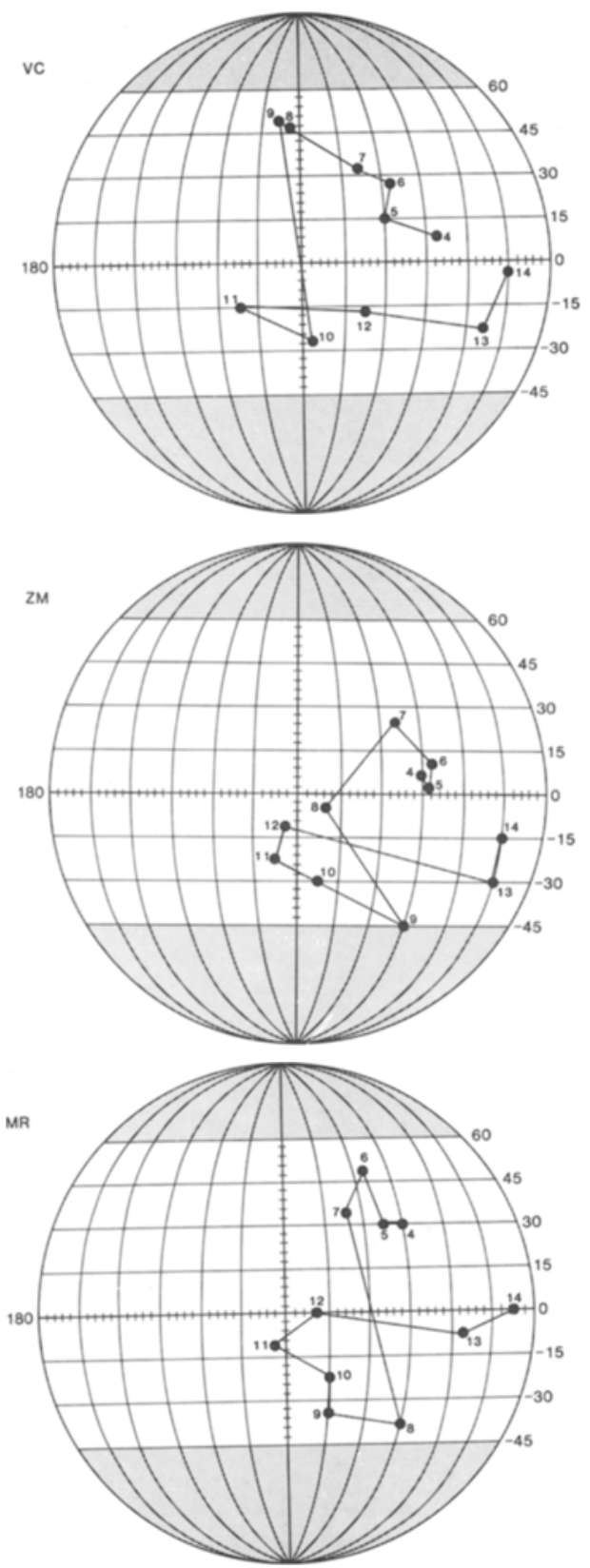

Figure 2. Means of the components of CPAs associated with 1.0$\mathrm{kHz}$-wide noise bands centered at $4.0-14.0 \mathrm{kHz}$ in steps of $1.0 \mathrm{kHz}$ for the 3 listeners shown in Figure 1.

tions of the mean CPAs for the other center frequencies ranging from 4.0 to $14.0 \mathrm{kHz}$. Plots of the mean CPAs, ranging from 4.0 to $14.0 \mathrm{kHz}$, for the other 2 listeners whose partial data are shown in the previous figure are also presented. For purposes of clarity, the spatial location of mean CPAs for center frequencies separated by steps of $1.0 \mathrm{kHz}$ rather than $0.5 \mathrm{kHz}$ are plotted. The maps of mean CPAs as a function of differently centered narrow noise bands for the remaining 3 listeners are qualitatively similar to those shown in Figure 2 in that the general pattern is for the mean of the CPAs associated with the narrow noise band to migrate upward and toward the rear as the center frequency of the noise band increases. At center frequencies of around 8.0 or $9.0 \mathrm{kHz}$, mean CPA associated with these noise bands drops to a lower elevation, and with further in- creases in the center frequency, mean CPA moves again somewhat farther toward the rear. When the center frequency reaches 13.0 or $14.0 \mathrm{kHz}$, the mean CPA associated with this frequency segment returns to the front. These results are in accord with the human data published by Middlebrooks et al. (1989), who also described, in terms of isolevel contours, an area of maximal amplification whose spatial pattern migrated in a similar fashion with increases in stimulus frequency. We also observed, in some instances, a split in the vertical location of the CPA when the center frequency was around $8.0 \mathrm{kHz}$ and a split in the horizontal location of the CPA when the center frequency was around $12.0 \mathrm{kHz}$. Since only the mean CPAs are plotted in Figure 2, the discontinuities within a CPA associated with a specific center frequency are not evident. Hence, for these few cases, the mean is not a representative index of the CPA's position. ${ }^{2}$

\section{Behavioral Measurements}

Selecting CPAs associated with "high" and "low" spatial regions. As stated in the introduction, we consider the CPA as the spatial referent of a given frequency segment. If there is a close concordance between the CPA (an area determined from a physical measurement) and a monaural location judgment, then monaural location judgments of different frequency segments should overlap with the CPAs associated with these frequency segments. On the basis of the CPAs calculated for each listener, we fabricated a stimulus that we expected that listener to judge consistently as originating above another "tailored" stimulus. We examined each subject's plot of mean horizontal-vertical coordinates of the CPAs as illustrated in Figure 2. From each plot, we chose a frequency segment whose associated CPAs were located above the CPAs associated with a different frequency segment within the same plot. We characterized the first frequency segment as linked to a CPA " "high" in space relative to the CPA associated with the other frequency segment, which we characterized as linked to a CPA "low" in space. We should emphasize that people differ with respect to the exact configuration of their pinna contours, which most likely accounts for the observation that for any given narrow noise band, the distribution of the associated CPAs among people also differs in detail. The basis for our selection of frequency segments whose CPA would be high and frequency segments whose CPA would, relatively speaking, be low can best be explicated through examples. Referring again to Figure 2, consider the mean CPA plot for Listener V.C. To establish a high CPA, we chose a 5.0-kHz-wide band of noise centered at $6.5 \mathrm{kHz}$. And for a low CPA, we chose a 4.0-kHz-wide noise band centered at $12.0 \mathrm{kHz}$. On the other hand, for Listener Z.M., we chose a 3.5-kHz-wide noise band centered at $5.75 \mathrm{kHz}$ as a stimulus whose referent in space, CPA, would be above that associated with a $5.5-\mathrm{kHz}$-wide noise band centered at $11.25 \mathrm{kHz}$, a stimulus whose referent in space would be low. In short, the bandwidth and center frequency were tailored to the listeners' plots of CPAs. To reiterate, labeling the CPAs as high or low in space simply means that the CPA associated with one frequency segment was located above, that is, higher than the CPA associated with a different frequency segment. Table 1 lists the bandwidths and their respective center frequencies for the high and low CPAs based on each listener's plot of CPAs.

Localization tests. Before testing, each listener was rendered monaural by the insertion of an ear defender (E-A-R) in his/her right ear and then covering that pinna with an earmuff (Bilsom Viking 2318). Next, thresholds were obtained for the noise bands fashioned for the particular listener-noise bands composed of frequencies whose CPAs were designated, on the basis of the microphone measurements, as high and those composed of frequencies whose CPAs were designated as low. It was not deemed feasible to measure the threshold for sounds emanating from each loudspeaker, as 104 of them were employed. Therefore, to save time and effort on the part of both listener and experimenter, thresholds of the sounds generated by only 9 of the loudspeakers were taken. 
Table 1

Bandpass Stimuli Based on Listeners' CPAs, Which Represented Their Upper and Lower Regions of Space

\begin{tabular}{lccccc}
\hline & \multicolumn{2}{c}{ Upper Spatial Region } & & \multicolumn{2}{c}{ Lower Spatial Region } \\
\cline { 2 - 3 } \cline { 5 - 6 } Listeners & BW & CF & & BW & CF \\
\hline M.R. & 3.0 & 5.5 & 6.0 & 11.0 \\
V.C. & 5.0 & 6.5 & 4.0 & 12.0 \\
Z.M. & 3.5 & 5.75 & 5.5 & 11.25 \\
M.U. & 5.0 & 6.25 & 7.0 & 11.5 \\
H.F. & 5.0 & 6.25 & 3.5 & 11.75 \\
C.O. & 4.5 & 6.25 & 4.0 & 11.0 \\
\hline
\end{tabular}

Note-BW signifies bandwidth (in kilohertz); CF signifies center frequency (in kilohertz).

These were stationed at $0^{\circ}, 90^{\circ}$, and $180^{\circ}$ in the horizontal plane; their vertical coordinates were $-45^{\circ}, 0^{\circ}$, and $60^{\circ}$. Thresholds for sound emanating from the remaining loudspeakers were estimated through interpolation.

A test session consisted of presenting a series of noise bursts from each of the 104 loudspeakers. The listeners were requested to identify the loudspeaker that was generating the stimulus. They were told that each loudspeaker was assigned a number in the horizontal plane and a number in the vertical plane. In the horizontal plane, numbers ranged from 1 through 13 for azimuthal positions from $0^{\circ}$ to $180^{\circ}$, respectively. In the vertical plane, numbers ranged from 1 through 8 for vertical positions from $-45^{\circ}$ through $60^{\circ}$, respectively. They were requested to report first the horizontal coordinate of the sound source, then the vertical coordinate. The listeners, after viewing the array of loudspeakers, expressed no difficulty in following our instructions. Each listener was also cautioned not to move his/her head during a test session; a headrest fixed to the back of his/her chair promoted compliance with our instructions. The experimenter was able to visually monitor the subjects through a small window to make certain that subjects were adhering to the nomovement edict. The sounds, 10-msec rise-fall time, 100-msec duration with an interstimulus interval of $300 \mathrm{msec}$, were presented at a sensation level of approximately $25 \mathrm{~dB}$ from measured and interpolated thresholds. Stimulus level varied by $+3,0$, and $-3 \mathrm{~dB}$ re $25 \mathrm{~dB} \mathrm{SL}$ reference level in order to discourage listeners from using relative loudness as a cue for the sound's source. Less intense sounds might be judged as originating from behind, notwithstanding the fact that spectral cues for front and rear would have been available irrespective of overall stimulus level (Asano, Suzuki, $\&$ Sone, 1990). To ensure that the test stimulus was inaudible to the right (blocked) ear, we compared thresholds when both ears were blocked with thresholds when neither ear was blocked. Blocking both ears elevated thresholds for all listeners by over $40 \mathrm{~dB}$. Obviously, the combination of the muff and ear defender effectively attenuated our stimuli, which consisted of frequencies $>3.5 \mathrm{kHz}$. For 3 listeners, the noise bands associated with CPAs representing their lower spatial regions were presented on the first test session; noise bands associated with CPAs representing their upper spatial regions were presented on the second session. The reverse held for the remaining 3 listeners. Delivering the stimuli in a block of either high or low associated CPAs rather than intermingling the respective stimuli eliminated the necessity of frequently manipulating the bandwidths and center frequencies. Testing time was shortened significantly, and reproducibility of stimulus characteristics was assured. Presentation order of sounds from the different loudspeakers was irregular, and the chain of noise bursts continued until the listener made a location judgment. No feedback was ever provided on the listeners' location judgments, nor had the listeners ever received feedback in previous localization studies. The frequency distribution of each listener's location judgments for each of the two different noise bands was entered on a $13 \times 8$ matrix, representing horizontal and vertical coordinates, respectively.
Selective filtering of differently centered narrow noise bands. On the basis of individual listeners' spatial distribution of their CPAs, we notched from a $3.5-\mathrm{kHz}$ highpass noise, a $2.0-\mathrm{kHz}$-wide noise band linked with CPAs whose spatial locations were either high, low, front, or back. For example, in the case of Listener M.R. (see Figure 2), we chose to center the $2.0-\mathrm{kHz}$-wide notch at $6.0 \mathrm{kHz}$ for the high location; at $9.0 \mathrm{kHz}$ for the low location; at $5.0 \mathrm{kHz}$ for the front location, and at $11.0 \mathrm{kHz}$ for the back location. In most instances, the choice of the center frequency of the notch filter (A.P. Circuit Model 403) for removing frequency segments whose associated CPAs occupied a high, a low, a front, and a back spatial region was straightforward. As a guide for setting the center frequency of the notch filter(s), the sound's spectrum was displayed on the screen of a spectrum analyzer (Hewlett-Packard Model $3580 \mathrm{~A}$ ) and the notch was visually centered at the desired frequency. To ascertain the characteristics of the notch when the stimulus emanated from our loudspeakers, we placed a B\&K $1 / 2$-in. microphone at a region normally occupied by the subject's head during the localization tests. The output of the microphone indicated a symmetrical notch, $2.0-\mathrm{kHz}$ wide at the $3-\mathrm{dB}$ downpoint (one half energy point), whose depth at center frequency was $15 \mathrm{~dB}$ when one filter was employed and $18 \mathrm{~dB}$ when two filters were arranged in series. The question here was, which CPA did we "remove" when we notched out a particular frequency segment. We adhered to the following procedure, which may best be conveyed by taking another example. Consider a $3.5-\mathrm{kHz}$ highpass noise in which a $2.0-\mathrm{kHz}$ notch was centered at $8.0 \mathrm{kHz}$. From our data on head-related transfer functions, we identified the CPA (location of loudspeakers that generated a microphone output within $1 \mathrm{~dB}$ of peak reading) for the $1.0-\mathrm{kHz}$-wide noise band centered at $7.5 \mathrm{kHz}$ and the CPA for the $1.0-\mathrm{kHz}$-wide noise band centered at $8.5 \mathrm{kHz}$, thereby covering the frequency segment extending from 7.0 to $9.0 \mathrm{kHz}$. By means of selective filtering, the spectral cues, which normally would have provided information on where the sound was coming from, would presumably be less effective when localizing the modified stimulus. As a consequence, we expected the listener to choose a loudspeaker from another spatial region as the genesis of the sound.

Localization tests. In the manner described earlier, monaural thresholds for a 3.5-kHz highpass noise band with and without the variously placed notches were obtained at the nine different loudspeaker locations; thresholds for the stimuli generated by the other loudspeakers were estimated through interpolation. During testing, the sounds were presented at approximately $25 \mathrm{~dB}$ SL re measured or interpolated thresholds. $A \pm 3-\mathrm{dB}$ level change was again intermingled among the localization trials. The presentation of stimulus conditions followed a $5 \times 5$ Latin-square arrangement where rows represented listeners, columns represented test sessions, and Latin letters represented differently tailored noise bands-four differently placed notches in the $3.5 \mathrm{kHz}$ highpass noise band, and the highpass noise band without a notch. A test session was composed of 104 localization trials, with each loudspeaker delivering, in a series of bursts, the scheduled noise band only once. The listeners were given only one test session per visit. This part of the study consisted of five test sessions - four differently filtered noise bands and the $>3.5-\mathrm{kHz}$ noise band. The entire series of localization tests was repeated using two notch filters, arranged in series, resulting in a notch deeper by $3 \mathrm{~dB}$ but centered at the same frequency. In all, each of the five noise stimuli was presented to a listener 208 times. Stimulus bursts were continued until a listener made a location judgment; no feedback was provided at any time during the course of the study. Each listener's distribution of location judgments for the $3.5-\mathrm{kHz}$ highpass noise and the four noise bands with differently placed notches were plotted separately on $13 \times 8$ matrices. By inspection, the distributions of a listener's location judgments for that notched stimulus were essentially the same regardless of whether one or two notch filters were used. Hence, we pooled the data. 


\section{RESULTS}

\section{Concordance Between the Distribution of Location Judgments and Distribution of CPAs in the Vertical Plane}

With few exceptions, listeners reported a noise band as originating within the CPA associated with that particular noise band. That is, each listener judged sounds whose frequency segments were associated with a CPA nested in the listener's upper region of space as originating above those sounds whose frequency segments were associated with a CPA nested in the listener's lower region of space. This result is illustrated in Figure 3. Here, the means of each listener's elevation judgments are shown for sounds whose frequency compositions were associated with the CPAs occupying the low and high regions of space. Standard error of the mean is represented at the top of each column. Without question, the frequency composition of the stimulus strongly influenced the elevation judgments. Differences between mean elevation judgments for the two sets of stimuli were highly significant $(p<.001)$ as revealed by paired $t$ tests.

The frequency segments were selected on the basis of a difference in the elevation of the associated CPAs, one CPA being located above the other in the vertical plane. They were not selected on the basis of a difference in the horizontal location of the associated CPAs, and as can be seen in Figure 2, CPAs distributed along the horizontal

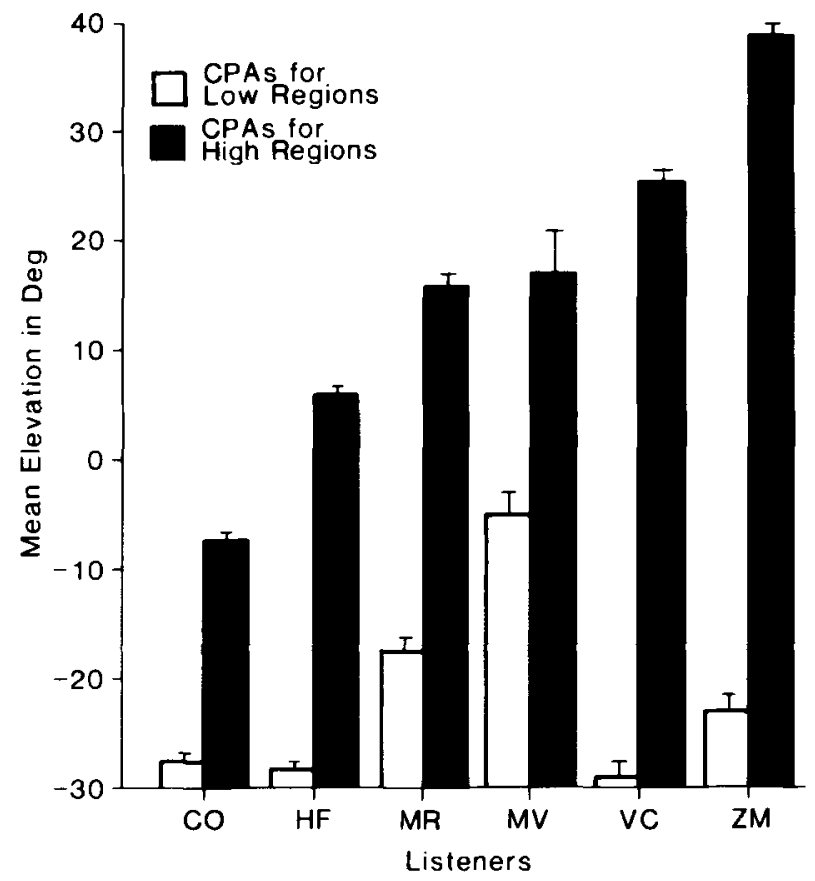

Figure 3. Mean elevation judgments in degrees for noise bands whose associated CPAs were "low" in the vertical plane (open columns) and whose associated CPAs were "high" in the vertical plane (filled columns). The standard errors of the respective means are shown above each column of the bar graph.

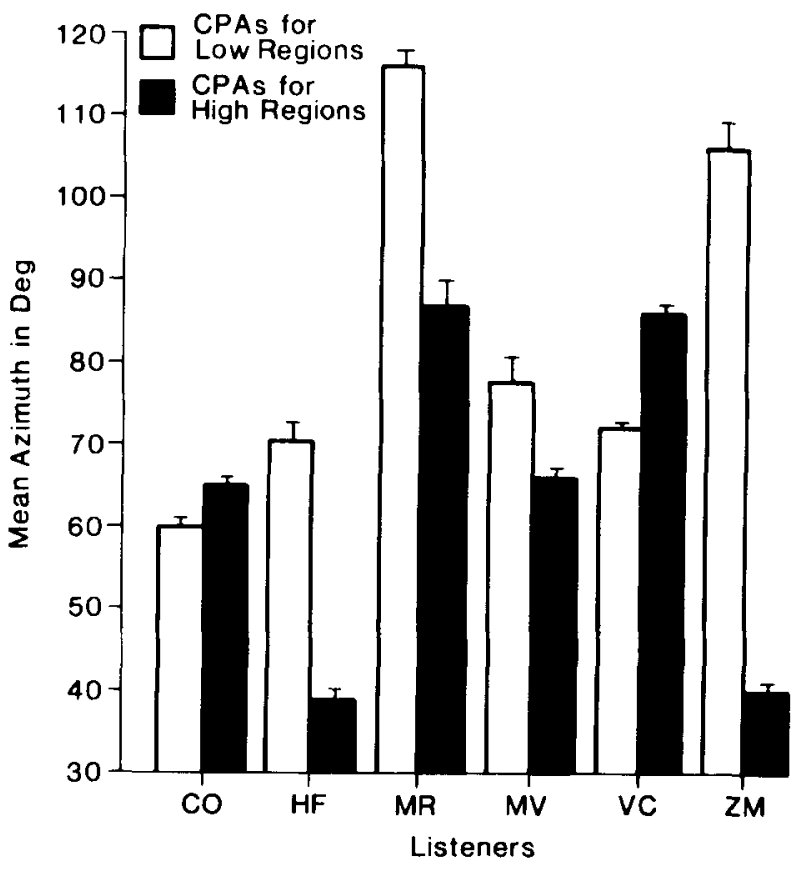

Figure 4. Mean location judgments in the horizontal plane for noise bands whose associated CPAs were "low" in the vertical plone (open columns) and whose associated CPAs were "high" in the vertical plane (filled columns). The standard errors of the respective means are shown above each column of the bar graph.

plane were linked to the frequency segments selected both for high and for low CPAs. As shown in Figure 4, the distribution of location judgments in the horizontal plane for the same frequency segments were not segregated, one in front of the other, in an orderly manner. The performance data for Listener Z.M., and to a somewhat lesser extent for Listener M.R., did, however, show a spatial separation of location judgments along the horizontal plane for the two different frequency segments; the frequency segments whose associated CPAs were low in the vertical plane were judged as originating to the rear of those whose associated CPAs were high (see Figure 4).

With respect to the relation between monaural location judgments of frequency segments whose CPAs were designated as high or low in space, it is informative to examine further the data of the 3 listeners featured in Figures 1 and 2 . The point is to compare, for each subject, the distribution of location judgments with the distribution of the CPAs associated with the different stimulus frequency segments that served as the stimuli. Their distribution of elevation judgments of frequency segments with high and low CPAs is plotted in the left panel of Figure 5; their distribution of azimuthal judgments for the same frequency segments is plotted in the right panel. The degree of concordance between location judgments of specific frequency segments (Figure 5) and the CPAs associated with these frequency segments can be detected by examining Figure 6. Here, the distribution of the CPAs associated with the respective frequency segments, listed 

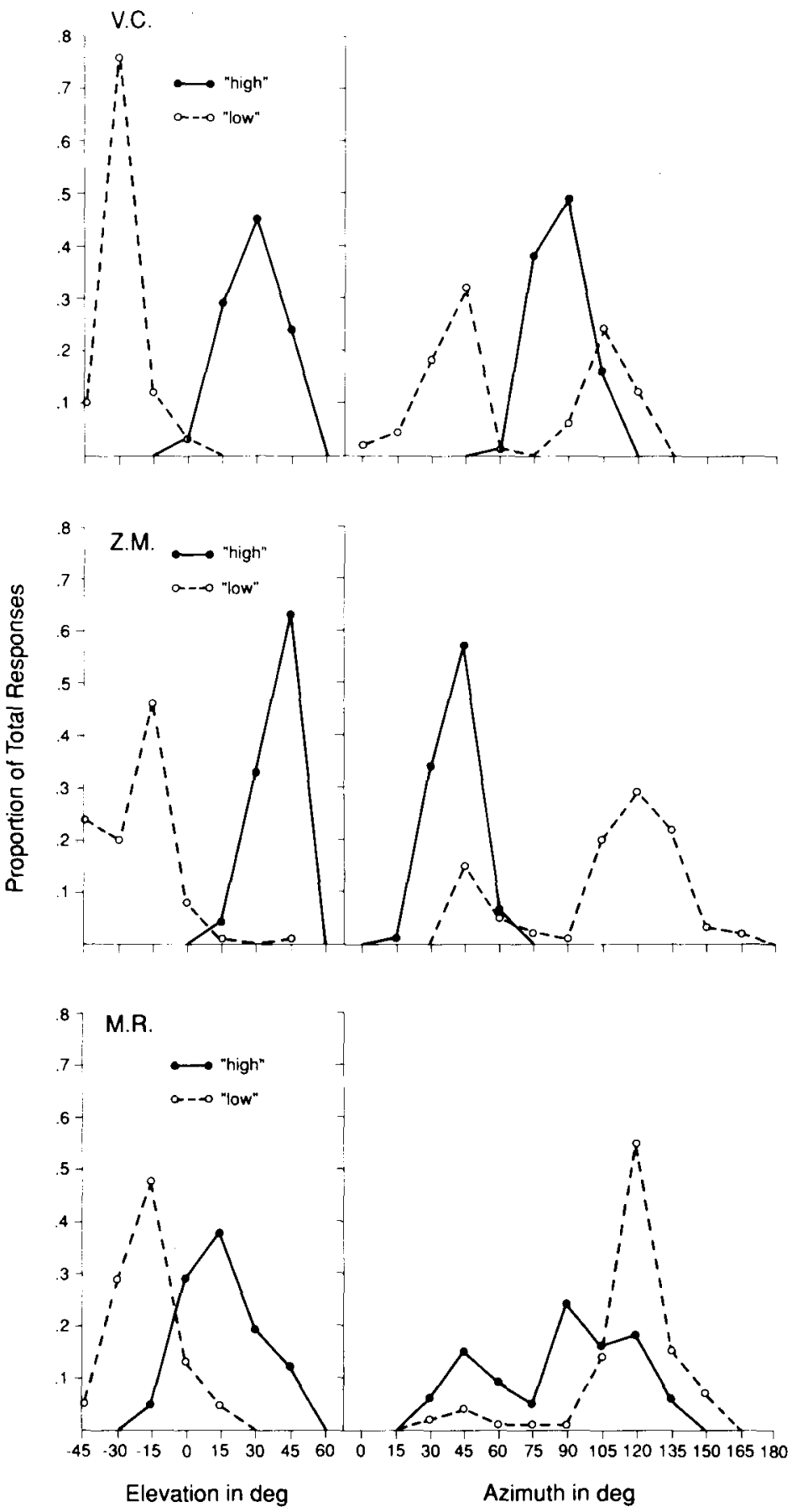

Figure 5. Distribution of location judgments when the stimulus contained frequencies whose CPAs were "high" in space (solid lines) and the stimulus frequencies whose CPAs were "low" in space (dashed lines). In the left panel, the proportion of total responses is plotted against elevation. In the right panel, the proportion of total responses is plotted against azimuthal position. The listeners are identified by their initials. 

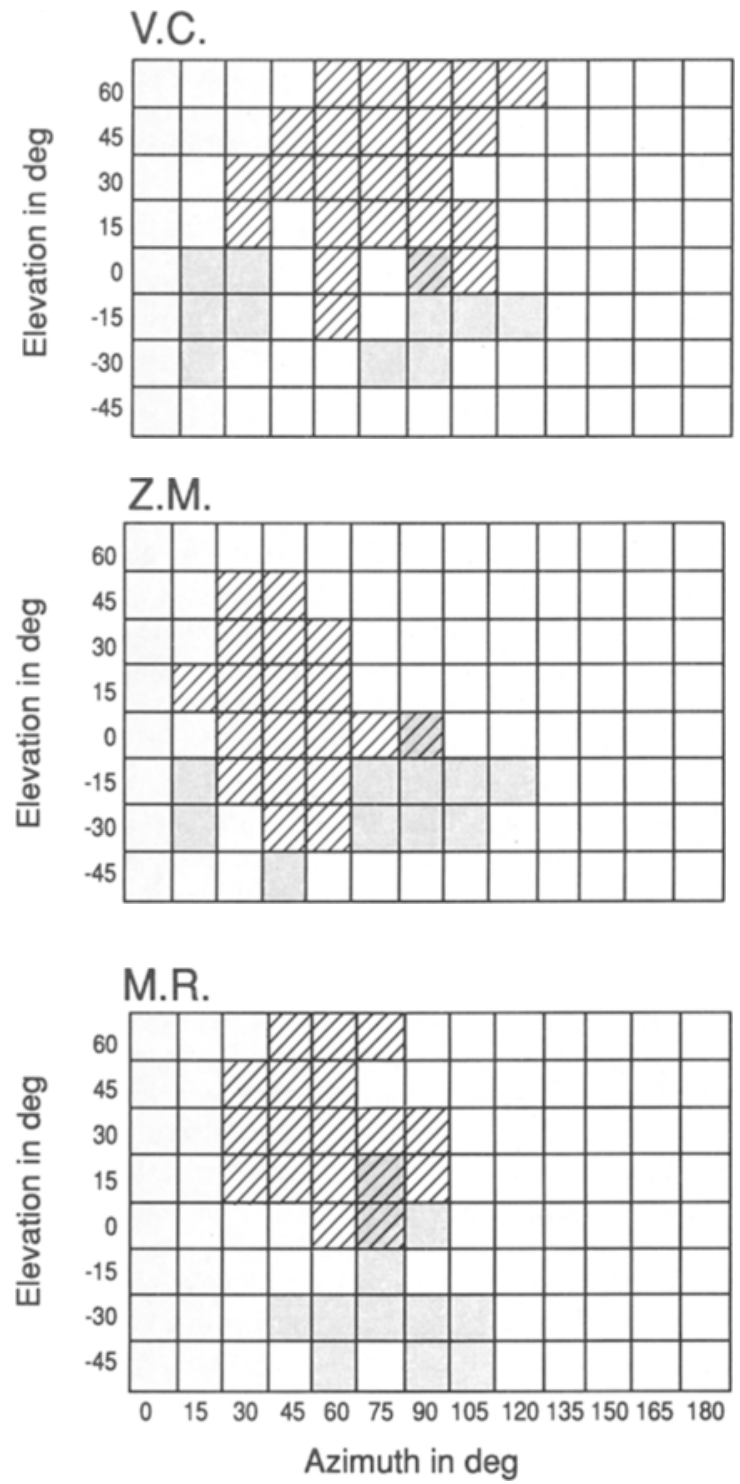

Figure 6. Distribution in two-dimensional space of CPAs associated with different frequency bands. For each listener, the CPA associated with one of the frequencies was designated as "high" (striped area) and the CPA associated with the other frequency band was designated as "low" (stippled area). The listeners are identified by their initials. in Table 1 , is plotted in two-dimensional space. The data for this plot were obtained by calculating the CPA for each differently centered frequency, separated by $1.0 \mathrm{kHz}$, of the narrow noise band contained in the wider frequency segment that served as the stimulus. So, for example, one of the stimuli that Listener V.C. was asked to localize was a $5.0-\mathrm{kHz}$-wide noise band centered at $6.5 \mathrm{kHz}-\mathrm{a}$ band containing frequencies ranging from 4.0 to $9.0 \mathrm{kHz}$. The CPAs for the $1.0-\mathrm{kHz}$-wide noise bands centered at 4.5 , $5.5,6.5,7.5$, and $8.5 \mathrm{kHz}$ were entered on a $13 \times 8$ matrix representing the 104 loudspeakers arrayed in the left hemifield. For Listener V.C., the combined CPAs associated with this noise band were designated as high and their distribution in two-dimensional space is represented by the striped squares. The other stimulus that Listener V.C. was asked to localize was $4.0 \mathrm{kHz}$ wide and centered at $12.0 \mathrm{kHz}-\mathrm{a}$ band containing the $1.0-\mathrm{kHz}$ wide noise bands centered at $10.5,11.5,12.5$, and $13.5 \mathrm{kHz}$. The combined CPA associated with this noise band was designated as low, and its distribution in twodimensional space is represented by the stippled squares. The several spatial locations where a CPA associated with one narrow noise band $(1.0 \mathrm{kHz}$ wide) overlapped with a CPA associated with one or more other narrow noise bands are not shown. The few instances of overlapping of CPAs between the two stimuli that Listener V.C. was requested to localize are indicated. Figures 5 and 6 illustrate that the distribution of elevation judgments by the 3 listeners is reasonably well separated with respect to high and low; so is the distribution of the CPAs associated with each stimulus. When considering these same stimuli in relation to their location judgments in the horizontal plane, the influence of the distribution of the CPAs associated with these stimuli, though less, can still be detected. For example, Listener V.C. judged the stimulus tailored to provide spectral cues for a high location to originate around $75^{\circ}$ and $90^{\circ}$ azimuth, which is near the center of the azimuthal distribution of the associated CPA. Listener V.C. judged the stimulus tailored to furnish spectral cues for a low location to originate either from the front or rear sector of the horizontal plane; accordingly, the azimuthal distribution of the CPAs associated with this stimulus was bimodal. The horizontal plane distribution of Listener Z.M.'s location judgments for the stimulus

Table 2

Number of Responses in Spatial Area of Interest When the Selected Frequency Segments Were Notched Out Compared with Number of Responses in the Same Area When No Filtering Was Administered

\begin{tabular}{lcccccc}
\hline & \multicolumn{7}{c}{ Listeners } \\
\cline { 2 - 7 } COND & M.R. & V.C. & Z.M. & M.U. & H.F. & C.O. \\
\hline Hi/Con & $22 / 35$ & $5 / 30$ & $22 / 116$ & $5 / 6$ & $7 / 13$ & $18 / 111$ \\
Lo/Con & $10 / 23$ & $22 / 39$ & $1 / 19$ & $1 / 9$ & $0 / 2$ & $4 / 21$ \\
Fr/Con & $20 / 38$ & $21 / 20$ & $49 / 112$ & $45 / 66$ & $21 / 40$ & $69 / 95$ \\
$\mathrm{Ba} /$ Con & $10 / 8$ & $14 / 25$ & $0 / 11$ & $11 / 26$ & $1 / 2$ & $10 / 24$ \\
Mean & $15.5 / 26$ & $15.5 / 28.5$ & $18 / 64.5$ & $15.5 / 26.8$ & $7.2 / 14.2$ & $25.2 / 62.8$ \\
\hline
\end{tabular}

Note-Hi, Lo, Fr, and Ba signify, respectively, high, low, front, and back locations of associated CPAs. Con signifies the control condition, that is, the $>3.5-\mathrm{kHz}$ noise band. COND signifies the experimental conditions being compared. 
fabricated to furnish spectral cues for a high location centered around $45^{\circ}$ azimuth; so did the distribution of the CPAs associated with this stimulus. Most of Z.M.'s horizontal plane location judgments for the stimulus tailored to provide spectral cues for a low location fell in the rear segment of the plane; a few fell in the front segment. The distribution of the CPAs associated with this stimulus reflect the location judgments. The distributions of Listener M.R.'s location judgments and the CPAs associated with the two different stimuli correlated less well with one another.

\section{Effect of Selective Filtering of Stimuli on Judgments of Source Location}

When specific frequency segments were attenuated by means of a notch filter, their effectiveness as spectral cues for location judgments was diminished. Documentation for this statement is based on the following observation: With the frequency distribution of location judgments when the $>3.5-\mathrm{kHz}$ noise band served as the control condition, the listeners less often reported a filtered stimulus as originating from the spatial referent (CPA) of a 2.0$\mathrm{kHz}$-wide frequency segment when that segment was attenuated by the notch filter. The data are summarized in Table 2. The number of location judgments toward high, low, front, and back when the relevant frequency segments were notched out is shown in the numerator. The denominator shows the number of location judgments falling within the same spatial regions for the unnotched noise. Our interest here was to test for statistical significance between the two sets of numbers recorded for the four spatial positions listed above. We separated the test sessions in which the frequency segment associated with the relevant CPA was notched out (treatment group) from the test sessions employing the $3.5-\mathrm{kHz}$ highpass noise (control group). We then fitted the following logistic regression model (see Hosmer \& Lemeshow, 1989; McCullagh \& Nelder, 1989) to the data and looked at the difference in number of location judgments in the relevant CPA between the treatment and control groups:

$$
\operatorname{logit}(p)=a+b F,
$$

where $p$ is the proportion of all trials for which the location judgments fell into the CPA, logit( $p)$ is the log odds ratio of $p$, which is defined as $\log p /-p, a$ is the intercept, $b$ is the slope (which was tested for its deviation from zero), and $F$ is a factor with two levels. Level 1 corresponds to the treatment group, and Level 2 corresponds to the control group. We first fitted four logistic regression models separately to four data sets, $\mathrm{Hi} / \mathrm{Con}, \mathrm{Lo} / \mathrm{Con}$, $\mathrm{Fr} / \mathrm{Con}$, and $\mathrm{Ba} / \mathrm{Con}$ (see Table 2 for the original data set). Then we combined these four data sets to fit another logistic regression model. A statistical package called GLIM (see Healy, 1988) was used to fit these five models. On the basis of five fitted logistic regression models, we calculated the Wald statistic, which is defined as the ratio of the estimated coefficient $(b)$ to its standard error $\left(S E_{b}\right)$. These results demonstrated that the differences in the number of location responses in the relevant CPA between the treat-
Table 3

Proportion of Location Judgments by Control Condition, Direction Toward the Spatial Regions Occupied by CPAs, for High, Low, Front, and Back When the Frequency Segments Associated with the Different CPAs Were Notched Out

\begin{tabular}{|c|c|c|c|c|}
\hline $\begin{array}{l}\text { Notched-Out } \\
\text { Segments }\end{array}$ & High & Low & Front & Back \\
\hline High & .25 & 2.51 & .72 & 2.26 \\
\hline Low & $1 . \overline{40}$ & .34 & 1.48 & .94 \\
\hline Front & .75 & .71 & .61 & 1.02 \\
\hline Back & 1.07 & .87 & 1.39 & .48 \\
\hline
\end{tabular}

ment group and the control group were significant in terms of the logit scale for all five comparisons. All $p$ values were less than .000025 . Clearly, filtering out the frequency segment associated with its CPA exerted a strong effect on the distribution of location judgments.

Table 3 provides partial information on the distribution of location judgments when a particular frequency segment associated with CPAs occupying high, low, front, and back were notched out; location judgments outside these designated spatial regions were not counted. Generally speaking, notch filter settings that affected location judgments normally directed to the high spatial regions (based on the frequency distribution of location judgments for the $>3.5-\mathrm{kHz}$ noise band) also affected location judgments normally directed to the front spatial regions, and the converse. Notch filter settings that affected location judgments normally directed to the low spatial regions (again based on the frequency distribution of location judgments for the $>3.5-\mathrm{kHz}$ noise band) also affected location judgments normally directed to the back spatial regions, and the converse. We point out that those spectral segments whose CPAs are high in space and those whose spectral segments are front in space are near one another in frequency. They are also separated in frequency from the spectral segments whose CPAs are low and the spectral segments whose CPAs are back in space. The spectral segments whose CPAs are low and those that are back in space are also near one another in frequency. In short, notching out a particular frequency segment reduces, to some extent, the number of location judgments directed to CPAs associated with nearby frequency segments. Not to lose sight of the main point, the number of location judgments directed to CPAs associated with the notched-out frequency segment was least of all. This is evidenced by the entries underlined in Table 3.

\section{DISCUSSION}

This study continued the quest to identify more precisely which aspect of stimulus frequency content influences the location judgments of monaural listeners. As the result of directional filtering by the pinna, different frequency segments of a complex sound are amplified more when the sound originated from one location as compared with all other locations. We have referred to these locations as CPAs, which were defined in the introduction. When the stimulus frequency composition was tailored so that the CPA for one stimulus was situated, in the vertical plane, 
above the CPA for the other stimulus, monaural judgments of elevation were distributed accordingly. Presenting a sound whose frequency composition possessed a CPA, or spatial referent, occupying an upper region of space was equivalent to filtering out the range of frequencies whose CPA occupied a lower region of space. This experimental technique was applied more selectively by means of notch filters. And as predicted, the listeners less often reported the sound as originating from its CPA after the frequency segment linked with this particular CPA was notched out. True, the listeners still reported sounds as occasionally emanating from the region in question, but this can be accounted for, in part, by the fact that components of CPAs linked to adjacent (nonfiltered) frequency segments spatially overlapped with CPAs linked to the frequency segment that was now notched out. In other words, notching a specific frequency segment does not remove all spectral cues associated with a particular spatial region. We should also emphasize that inserting a $2.0-\mathrm{kHz}$-wide notch in the wide noise band reduces, but does not abolish, the influence of that specific frequency segment chosen for evaluation of its effect on localization performance.

Our success in finding a concordance between the spatial referent of a frequency segment determined through physical measurements and the spatial referent of the same frequency segment determined through behavioral measurements marks the positive aspect of this investigation. On the basis of the physical measurements, which provided the CPAs associated with different frequency segments, we were able to predict with a high level of statistical significance where (and where not) listeners would direct their location judgments. There is a multitude of reasons for our failure to demonstrate an even tighter linkage between the two sets of measurements. To name a few: As referred to earlier, the CPAs associated with some frequency segments showed a discontinuity in space. Also, setting a 1-dB boundary for a CPA of a given 1.0-kHz-wide frequency segment was arbitrary; choosing a cutoff point greater than or less than $1 \mathrm{~dB}$ of maximum amplitude might establish a closer link between the physical and behavioral measurements. Furthermore, there was variability among sets of sound-pressure measurements taken at the ear canal entrance, and our test room, although sound deadened, is not anechoic. And last, without feedback, the listeners' attention to detailed spectral cues may flounder, and cognitive strategies that the listeners, in all likelihood, devise to cope with this demanding perceptual task may influence location judgments in unpredictable ways.

We were not concerned in this study with whether monaural listeners correctly identified the loudspeaker that was generating the sound or how close they were to being correct in their judgments. When the distribution of location judgments is biased via selective filtering, which certainly was the case in this study, the number of correct responses or the size of the error in localizing the sound is a misleading indicator of performance proficiency. For example, notching out a frequency segment whose associated CPA is high in space results in a greater concentra- tion of location judgments directed to regions low in space (see Table 3). This increase in the frequency of location judgments directed to the lower spatial regions increased the probability of obtaining more correct responses for the sounds that did, indeed, emanate from the lower spatial regions. Obviously, the listeners did not suddenly become more proficient at localizing sounds coming from the lower spatial regions.

Our main aim in this investigation was to find out whether the distribution of the listeners' location judgments would be in accord with the predictions based on the linkage between stimulus frequency and its associated CPA. We believe it most improbable that the relation between stimulus frequency content and perceived location will tum out to be an epiphenomenon. Butler (1971) suggested that the spatial referent of stimulus frequency reflects the operation of a mechanism that is tied in with the tonotopic organization of the central auditory nervous system. The pinna, while providing spectral cues for localization of broadband stimuli, is not responsible for the spatial referent of a narrow noise band. As Blauert (1983, pp. 111112) pointed out, the pinna (and head) only influence the level of a narrow noise band recorded at the ear canal entrance; it does not differentially affect the frequency content within the band. Indeed, the apparent location of tonal stimuli heard monaurally is, by and large, unaffected by pinna distortion (Butler, 1971). The fact that stimulus frequency possesses a referent in space has more general implications. Specifically, visual space is topographically represented from retina to visual cortex, and somatic space is topographically represented from the surface of the skin to the somatic cortex. In the auditory system, it is frequency that is organized systematically, and this organization is tonotopic extending from the cochlea to the auditory cortex. Since different tonal frequencies have different referents in space, it follows that auditory space, insofar as the frequency content of the sound plays a role, is also topographically represented in the auditory nervous system.

To return to the issue raised at the beginning of this paper: There is no question that learning is critical for attaining accuracy in monaural localization. With the use of feedback, monaural listeners can proficiently localize sound originating throughout the horizontal plane, an accomplishment that is not reached by untrained listeners (Butler, 1987). The question is, what is being learned? We suggest that in monaural localization of broadband sound, the auditory system detects the frequency segment that, because of the directional filtering properties of the pinna, is amplified more at that location than it is when the sound emanates from any other location. The spatial referent of this frequency segment influences the location judgment. We should emphasize that spatial referents of differently centered narrow-band noise occupy a relatively restricted portion of the hemifield, as evidenced in Figure 2. Perhaps that which primarily is acquired through training is the utilization of spectral cues (furnished by torso, shoulder, head, and pinna) for sounds originating outside the area encompassing the spatial referents of tonal frequen- 
cies, that is, the region on and around the median sagittal plane. Spectral cues may not be important for localizing sounds in these central areas since, under ordinary listening conditions, they are well served by binaural difference cues.

\section{REFERENCES}

Asano, F., Suzukı, Y., \& Sone, T. (1990). Role of spectral cues in median plane localization. Journal of the Acoustical Sociery of America, 88, $159-168$.

BlAUERT, J. (1969). Sound localization in the median plane. Acoustica, 22, 205-213.

Blauert, J. (1983). Spatial hearing. Cambridge: MIT Press.

Butler, R. A. (1971). The monaural localization of tonal stimuli. Perception \& Psychophysics, 9, 99-101.

ButLER, R. A. (1987). An analysis of the monaural displacement of sound in space. Perception \& Psychophysics, 41, 1-7.

Butler, R. A., \& Flannery, R. (1980). The spatial attributes of stimulus frequency and their role in monaural localization of sound in the horizontal plane. Perception \& Psychophysics, 28, 449-457.

Carlile, S. (1990). The auditory periphery of the ferret: 2 . The spectral transformations of the external ear and their implications for sound localization. Journal of the Acoustical Society of America, 88, 2196-2204.

DURlach, N. I., \& Colburn, H. S. (1978). Binaural phenomena. In E. C. Carterette \& M. P. Friedman (Eds.), Handbook of perception (Vol. 4, pp. 365-466). New York: Academic Press.

Healy, M. J. R. (1988). GLIM: An introduction. Oxford: Clarendon.

Hebrank, J., \& Wright, D. (1974). Spectral cues used in the localization of sound on the median plane. Journal of the Acoustical Society of America, 56, 1829-1834.

Hosmer, D. W., \& Lemeshow, S. (1989). Applied logistic regression. New York: Wiley.

Humanski, R. A., \& Butler, R. A. (1988). The contribution of the near and far ear toward localization of sound in the sagittal plane. Journal of the Acoustical Society of America, 83, 2300-2310.

MCCullagh, P., \& Nelder, J. A. (1989). Generalized linear models. London: Chapman \& Hall.

Middlebrooks, J. C., Makous, J. C., \& Green, D. M. (1989). Directional sensitivity of sound-pressure levels in the human ear canal. Journal of the Acoustical Society of America, 86, 89-108.

Musicant, A. D., \& Butler, R. A. (1984). The psychophysical basis of monaural localization. Hearing Research, 14, 185-190.

Musicant, A. D., Chan, J. C. K., \& Hind, J. E. (1990). Directiondependent spectral properties of cat external ear: New data and crossspecies comparisons. Journal of the Acoustical Society of America, 87, 757-781.

Pratt, C. C. (1930). The spatial character of high and low tones. Journal of Experimental Psychology, 13, 278-285.

Roffler, S. K., \& BUTLER, R. A. (1968). Localization of tonal stimuli in the vertical plane. Journal of the Acoustical Society of America, 43, 260-266.

\section{NOTES}

1. Humanski and Butler (1988) showed graphically (their Figure 2) the defining feature of a covert peak associated with a specific high frequency $(1.0-\mathrm{kHz}$-wide noise band centered at $11.0 \mathrm{kHz})$. The fact that the response amplitude to that specific frequency composition was critically dependent on source location was completely masked when response amplitude was plotted against frequency composition-the usual way of plotting frequency response curves.

2. It has been our experience that when listeners are required to localize narrow noise bands centered at these transition frequencies, some report the sound as coming from two different places, with one of the places predominating as the test progresses.

(Manuscript received August 20, 1991; revision accepted for publication May 3, 1992.) 